Provided for non-commercial research and education use. Not for reproduction, distribution or commercial use.

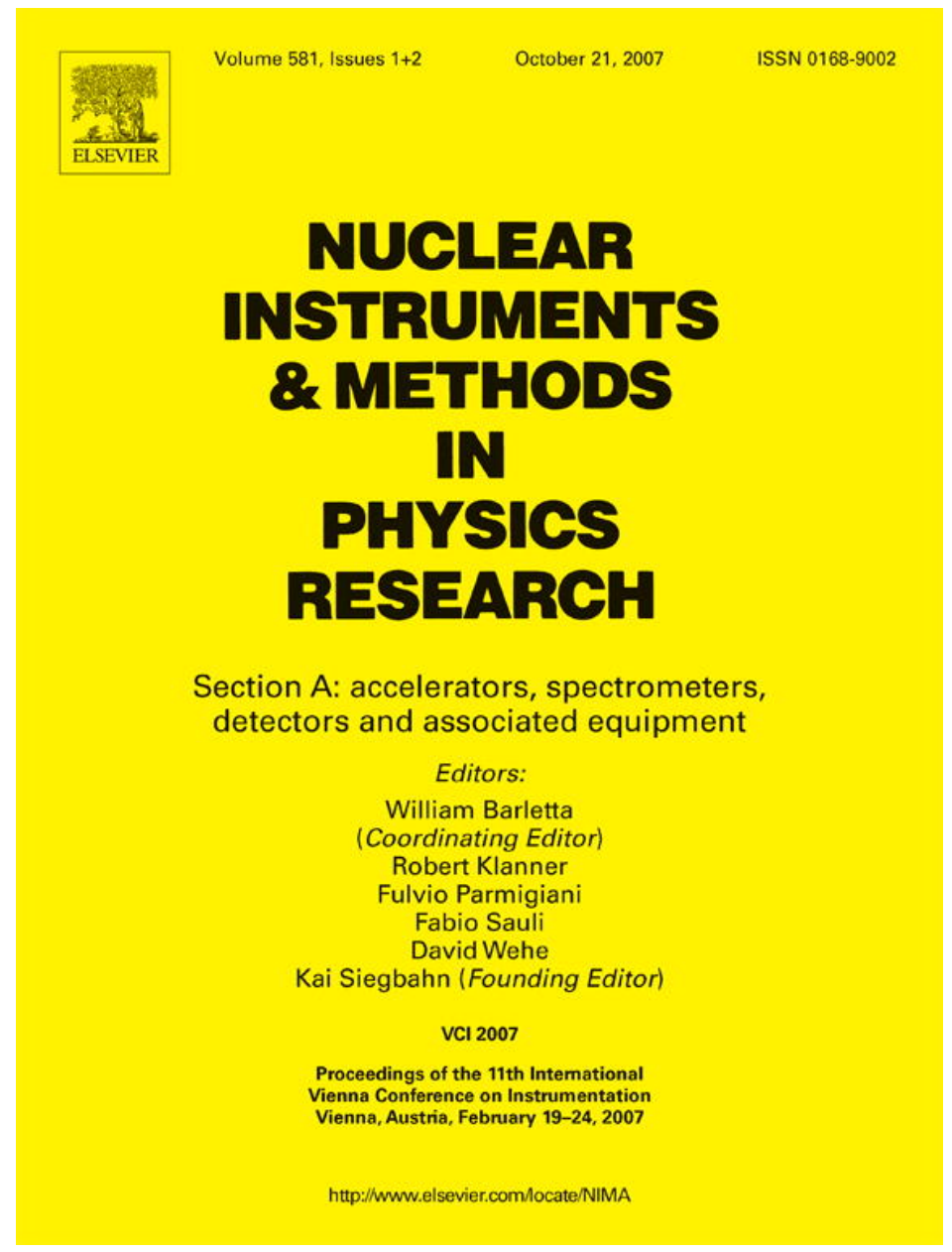

This article was published in an Elsevier journal. The attached copy

is furnished to the author for non-commercial research and education use, including for instruction at the author's institution, sharing with colleagues and providing to institution administration.

Other uses, including reproduction and distribution, or selling or licensing copies, or posting to personal, institutional or third party websites are prohibited.

In most cases authors are permitted to post their version of the article (e.g. in Word or Tex form) to their personal website or institutional repository. Authors requiring further information regarding Elsevier's archiving and manuscript policies are encouraged to visit: 


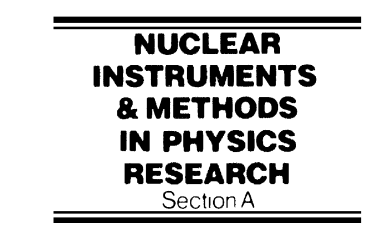

www.elsevier.com/locate/nima

\title{
Two-dimensional detector of thermal neutrons
}

\author{
V. Andreev, G. Ganzha, D. Ilyin, E. Ivanov, S. Kovalenko, \\ A. Krivshich*, A. Nadtochy, V. Runov \\ Petersburg Nuclear Physics Institute, Russian Academy of Science, 188300 Gatchina, Leningrad District, Russia
}

Available online 26 July 2007

\begin{abstract}
A two-dimensional detector of thermal neutrons has been designed and constructed for neutron diffraction experiments at the St. Petersburg Nuclear Physics Institute. It is based on a multiwire proportional chamber (MWPC) with cathode strip delay line readout and has a sensitive area of $170 \times 300 \mathrm{~mm}^{2}$ and anode wire spacing is $4 \mathrm{~mm}$. It operates with a gas mixture of $1.5 \mathrm{bar}{ }^{3} \mathrm{He}+2 \mathrm{bar} \mathrm{CF}_{4}$. To improve the gas purity by a few orders of magnitude, a new technology for fabrication of the detector's electrodes has been developed. An intrinsic resolution of $0.6 \mathrm{~mm}$ (FWHM) and a differential nonlinearity of $\pm 5 \%$ are achieved. It was shown that the detector, whose efficiency is about $60 \%$ for $9 \AA$ neutrons, has a resolution of $2.5 \mathrm{~mm}$ along the fine axis and about $4 \mathrm{~mm}$ for the perpendicular discrete axis. The dependence of the measured pulse height spectra from the applied high voltage and the electric field in the drift regions has been investigated. It turns out that for thermal neutrons the measured spectra are very similar to those obtained with proportional neutron counters filled with 10 bar ${ }^{3} \mathrm{He}$.
\end{abstract}

(C) 2007 Elsevier B.V. All rights reserved.

PACS: $28.20 . \mathrm{Fc} ; 29.40 . \mathrm{Cs} ; 29.40 . \mathrm{Gx}$

Keywords: Thermal neutron detector; Outgasing

\section{Introduction}

Multiwire proportional chambers filled with a ${ }^{3} \mathrm{He} / \mathrm{CF}_{4}$ gas mixture and delay line readout are widely used for thermal neutron detection [1-3]. The purpose of this work is to present the solution of a set of strongly interrelated design parameters to satisfy detector performance criteria. It is shown that in the framework of detailed consideration of each parameter finally both good spatial resolution, high efficiency, as well as differential and integral linearity, are obtainable.

In order to guaranty a reliable and optimal operation of the neutron detector, a wide range of scientific and technological investigations have been carried out:

- To improve and to optimize the detector mechanical characteristics a mathematical simulation of the detector behavior under pressures up to 10 bar was performed.

\footnotetext{
*Corresponding author. Tel.: + 78137146042 ; fax: + 78137137976.

E-mail address: kriv@pnpi.spb.ru (A. Krivshich).
}

- To minimize detector outgasing a new technology for fabrication of the detector's electrodes was developed. It opened a possibility to improve the gas purity by a few orders of magnitude.

- The detector gas sealing technology has been improved, so as to minimize the gas leak down to a level of less than $0.03 \%$ per day.

\section{Detector design}

\subsection{Thermal neutron detection and gas mixture}

Neutrons were detected by capture in ${ }^{3} \mathrm{He}$ gas according to the nuclear reaction:

${ }^{3} \mathrm{He}+\mathrm{n}^{-} \rightarrow \mathrm{p}+{ }^{3} \mathrm{H}+764 \mathrm{keV}$.

The problem of achieving the highest possible detector efficiency was one of the first to be solved during detector construction. From this point of view, ${ }^{3} \mathrm{He}$ is a very 
convenient gas for use because it has a very large capture cross-section for thermal neutrons $(26,500 \mathrm{~b}$ for $9 \AA$ neutrons). In our calculations, we considered design characteristics of the chamber, namely, the inevitable neutron absorption in an entrance window and in a gas gap between the entrance window and the first drift cathode (Fig. 1). The efficiency can be calculated as $\varepsilon=$ $\exp \left(\mu_{\mathrm{W}} d_{\mathrm{W}}\right) \times \exp \left(\mu_{\mathrm{G}} d_{\mathrm{G}}\right) \times\left[1-\exp \left(\mu_{\mathrm{D}} d_{\mathrm{D}}\right)\right]$, where $\mu=n \sigma$, with gas atom concentration $n$, neutron absorption crosssection $\sigma$, thickness of entrance window $d_{\mathrm{W}}$, gap distance between entrance window and cathode $d_{\mathrm{G}}$, and thickness of the active detector area $d_{\mathrm{D}}$. So, the expected overall detector efficiency should be about $60 \%(9 \AA)$ for a ${ }^{3} \mathrm{He}$ gas pressure of 1.5 bar.

The physical limit of the detector position resolution is dominated by the particle range from reaction (1) and their ionization losses. The proton and the triton are emitted in opposite directions from the locus of the nuclear reaction, and the emission is isotropic. The centroid of the ionization charge is displaced with respect to the nuclear reaction locus due to different ionization losses of proton and triton. The loci of the centroids for many neutrons are uniformly distributed on the surface of a sphere. The resulting probability distribution in any direction is a rectangular one. According to Ref. [1], the spheroid diameter $D_{\mathrm{sph}}=0.7 R_{\mathrm{p}}$, where $R_{\mathrm{p}}$ is the proton range. ${ }^{3} \mathrm{He}$ has low stopping power and that is the reason why $\mathrm{CF}_{4}$ has been used as a stopping gas in order to reduce the ranges. Hence, the gas limit to the spatial resolution is about $1.4 \mathrm{~mm}$ for our choice of 2 bar pressure of $\mathrm{CF}_{4}$.

\subsection{Detector construction}

Mathematical models of several variants of the mechanical design of the neutron detector have been carried out by means of FEM (finite-element method). The most successful design is chosen from the point of view of minimal deflection of an entrance window under internal pressure.

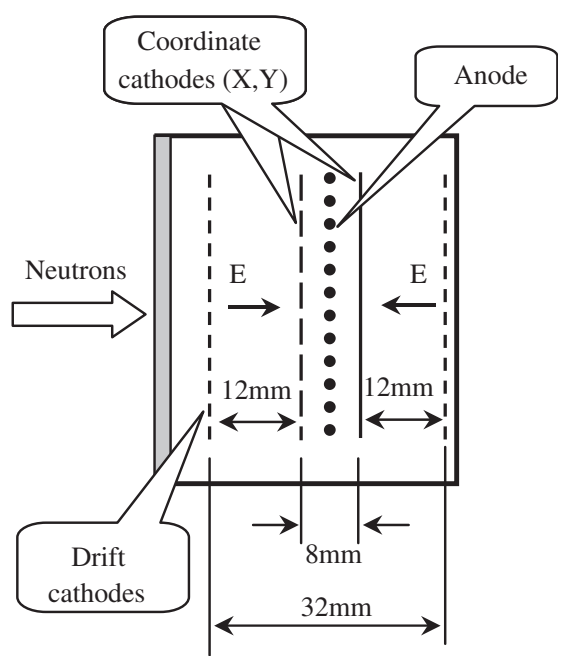

Fig. 1. Schematic view of the detector cross-section.
The chamber prototype was constructed from aluminum alloy $\mathrm{AlMg}_{5}$. This alloy has the most suitable combination of good mechanical strength and good neutron transmission properties. Moreover, it was measured that this material exhibits minimal neutron small-angle scattering in comparison with other investigated materials intended for detector construction. Some recommendations for an optimization of the chamber design have been accumulated during our investigations. This experience influences the design of the new detector $\left(300 \mathrm{~mm} \times 300 \mathrm{~mm}^{2}\right)$ that is currently under construction.

It was desirable to avoid a deflection of an entrance window under due to the gas pressure, since this could serve as a reason of position heterogeneity of the detector efficiency. After repeated tests and design debugging of the chamber it was possible to reduce the deflection of the entrance window (thickness $10 \mathrm{~mm}$ ) down to a minimum of about $0.2 \mathrm{~mm}$.

Because of the use of expensive ${ }^{3} \mathrm{He}$, special attention was given during detector design to the reduction of gas leakage from the chamber volume by improving the quality of the gas sealing. To evaluate the final value of this parameter, the temperature and the pressure of a gas mixture were measured in the chamber for a long time (about 400 days). The variation with time of the relative density of the gas mixture has been calculated. It was shown that the gas leakage rate was about $0.03 \%$ per day.

The detector design is essentially based on a conventional multiwire proportional chamber (Fig. 1) with two orthogonal cathode grids, located symmetrically about the central anode grid. The anode consists of gold-plated tungsten wires with a diameter of $25 \mu \mathrm{m}$ and a spacing of $4 \mathrm{~mm}$. The "anode-cathode" distance is also $4 \mathrm{~mm}$. The cathode wires have $55 \mu \mathrm{m}$ diameter and a step of $1 \mathrm{~mm}$. Each of the four wires are connected together in one strip that is connected to the delay line (impedance $100 \Omega$ ), with a specific delay of $6 \mathrm{~ns}$ per strip. So, the number of strips was: 78 (discrete axis) and 39 (fine resolution axis). In order to increase the detector efficiency there are two $12 \mathrm{~mm}$ absorption/drift regions, which are associated with each cathode of the MWPC. Thus, the active detector volume has a thickness of $32 \mathrm{~mm}$.

In order to achieve the best physical parameters of the detector it was necessary to optimize the anode signal transmission into the cathode strips. For this purpose, some more important geometrical parameters of the MWPC have been chosen according to the following principle: anode wire spacing, "anode-cathode" gap and strip width all have to be the same.

\subsection{Design of electrodes}

To reveal the long-term performance of the detector and to prevent degradation of the energy resolution, it is necessary to maintain a high level of gas mixture purity. This means that the detector has to be fabricated from materials that can be pumped to high vacuum and heated 
up to at least $100{ }^{\circ} \mathrm{C}$. To minimize detector outgasing, we started the development of a new technology for the fabrication of detector's electrodes and successfully finished this not too simple task. Now all electrodes are fabricated from quartz-glass that can be pumped to high vacuum and heated up to $130{ }^{\circ} \mathrm{C}$. The properties of the deposited printed circuits stay constant even after heating of the frames up to $150{ }^{\circ} \mathrm{C}$. Wire soldering can be done many times by soldering with a melting point of $280^{\circ} \mathrm{C}$. This has opened a way to improve the gas purity by a few orders of magnitude, since no glue has been necessary for the assembly of the detector.

\section{Experimental results}

\subsection{Detector efficiency}

In order to measure the detector efficiency, a reference proportional counter (filled with 10 bar ${ }^{3} \mathrm{He}$ ) normally intended for thermal neutron detection with an efficiency of practically $100 \%$ has been used. The new detector and the standard proportional counter were irradiated independently by collimated neutron beams (neutron flux density: $1.7 \times 10^{4} \mathrm{~s}^{-1} \mathrm{~cm}^{-2}$ ). From the counting rate difference, an efficiency of about $\varepsilon=60 \%$ was inferred, in good agreement with the result of the theoretical calculation (see Section 2.1).

\subsection{Differential nonlinearity}

The differential nonlinearity is defined as the derivative $\mathrm{d} x_{\mathrm{m}} / \mathrm{d} x$ of the position calibration curve $x_{\mathrm{m}}=f(x)$, where $x_{\mathrm{m}}$ is the measured position and $x$ is the true position. A measure of the differential nonlinearity is coming from the flat response of an uniform illumination spectrum. Any nonuniformity of the detector efficiency will show up in this spectrum. Fig. 2 shows a uniform illumination spectrum obtained by a wide beam of thermal neutrons along the anode wires ( $X$-axis). Differential nonlinearity for these results is below $\pm 5 \%$. This parameter is very sensitive for boundary discontinuities between two multipin connectors which are taking the signals from cathode

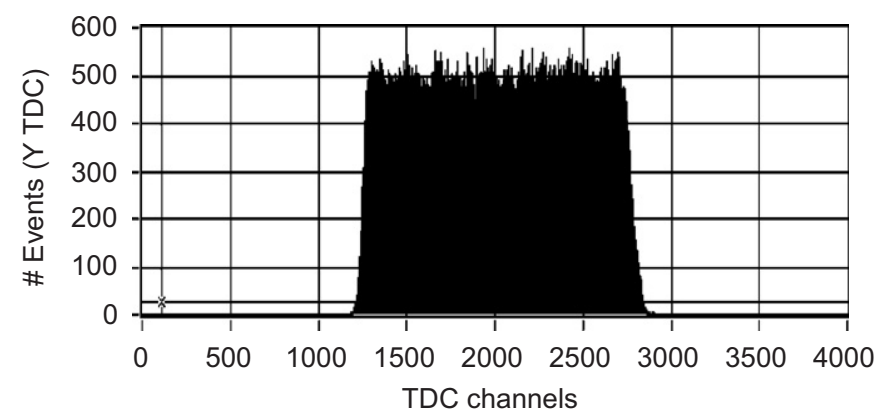

Fig. 2. Uniform illumination spectrum along the fine resolution axis (parallel to the anode wires). strips. There is no indication of discontinuities at the section boundaries.

\subsection{Position readout in the direction perpendicular to the anode wires ( $Y$-axis)}

As one can see from the differential nonlinearity measurement presented in Fig. 2, no edge effects have been observed in these spectrums, which usually manifest themselves as a rising up of local intensity at the edges of these spectra. It means that the absolute position error in this $X$-direction at the minimum and maximum of the uniform illumination response is low.

Fig. 3 shows the uniform illumination spectrum as seen in $Y$-direction. One can see well-shaped independent peaks, whose modulation has a period identical to the anode wire spacing. Because the peak positions are determined by the gas avalanches surrounding each anode wire, it allowed us to evaluate that the integral nonlinearity along the $Y$-direction is less than $0.4 \%$.

Generally speaking, it is possible to state that the absolute position error in both $X$ - and $Y$-directions at the minimum and maximum of the uniform illumination response is low and noticeably less than the spatial resolution dictated by physical reasons.

It is essential to notice that the width (FWHM) of these peaks determines the "interesting" resolution of detector,

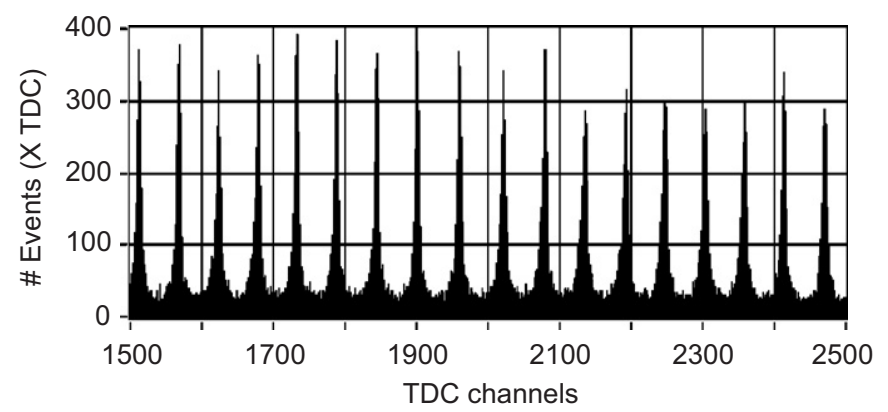

Fig. 3. Uniform illumination spectrum along the discrete axis (perpendicular to the anode wires).

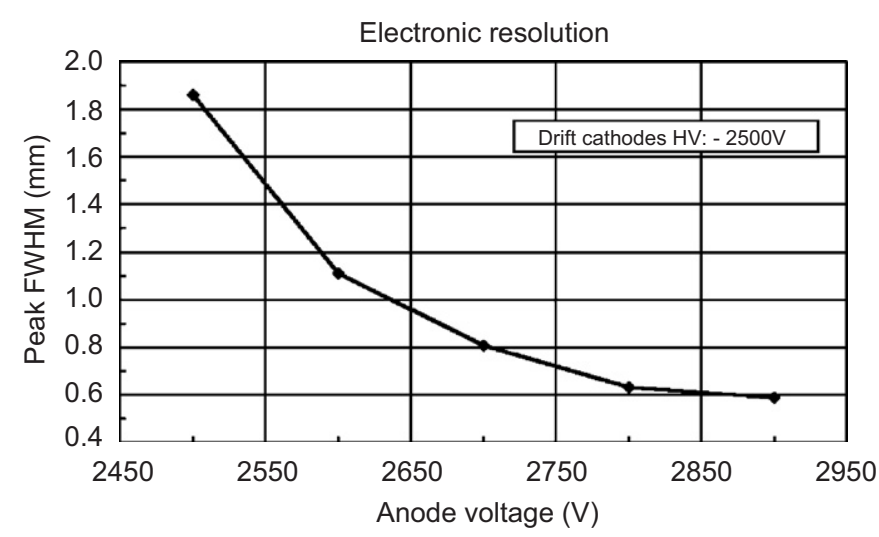

Fig. 4. Intrinsic resolution of detector via high voltage. 
which includes the intrinsic resolution due to electronic noise, delay line quality, electric field structure, etc. Obtained results are presented in Fig. 4. As one can see, the intrinsic resolution of detector is $0.6 \mathrm{~mm}$, which is a few times less than the physical gas limit.

\subsection{Position resolution}

To measure the spatial resolution, the detector has been irradiated by a narrow neutron beam whose width was discretely changed in a range from 0.5 to $4.0 \mathrm{~mm}$ by $\mathrm{Cd}$ collimators. A typical view of obtained spectra is shown in Fig. 5. As one can see from the measured data (Fig. 6), the spatial resolution of the detector is about $2.5 \mathrm{~mm}$ (FWHM). This is in good agreement with the theoretically expected resolution.

\subsection{Energy spectra}

The pulse height spectra behavior via anode high voltage and electric field tension in the drift regions has been investigated. It turned out that these spectra (Fig. 7a) are very similar to those obtained from the reference proportional counter, which has a gas pressure of 10 bar and looks like a "black body" for thermal neutrons (Fig. 7b).

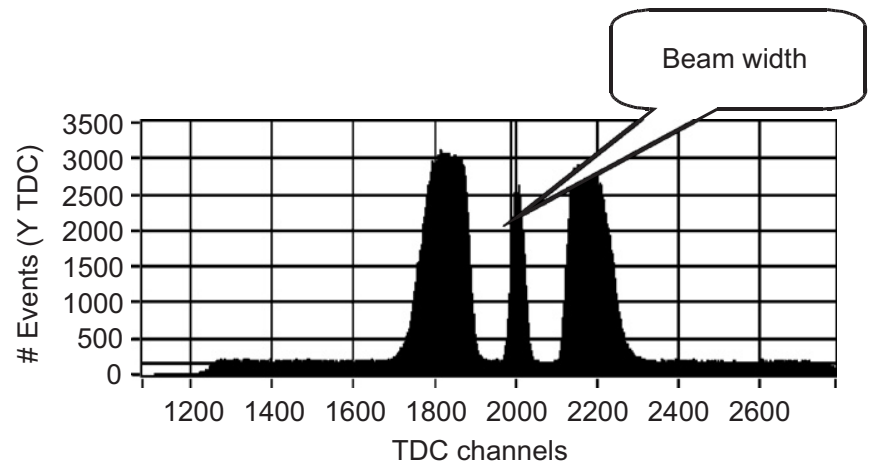

Fig. 5. Neutron position response measurement along the fine resolution axis.

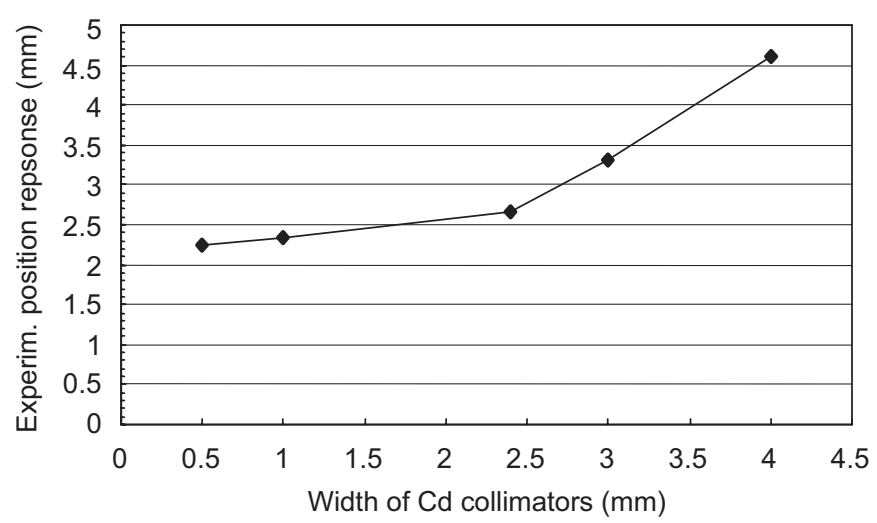

Fig. 6. Spatial resolution measurement.

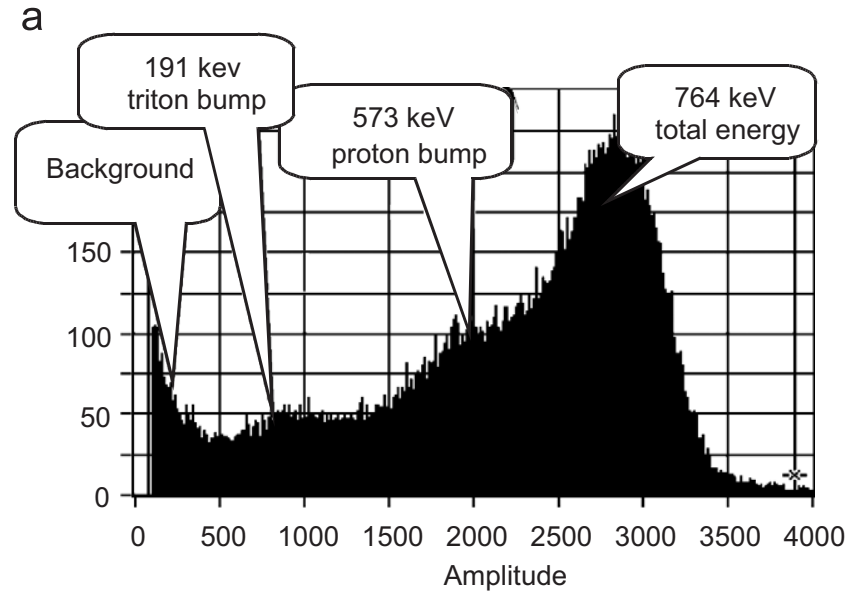

b

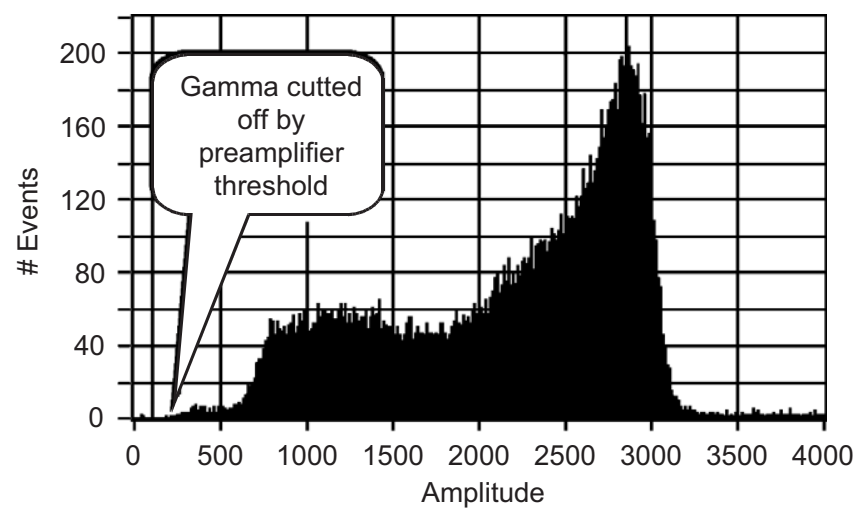

Fig. 7. (a) Energy spectrum obtained from the neutron detector (anode $\mathrm{HV}=2700 \mathrm{~V}$; drift region $\mathrm{HV}=-2500 \mathrm{~V}$ ). (b) Energy spectrum obtained from the reference neutron proportional counter $(\mathrm{HV}=-2250 \mathrm{~V})$.

As one can see from Fig. 7a, the energy resolution achieved in our new detector is very similar to those observed in the reference proportional counter. The energy spectrum exhibits a well-defined peak, which corresponds to the energy liberated in reaction (1). There are also bumps corresponding to the proton energy $(573 \mathrm{keV})$ and that of the triton $(191 \mathrm{keV})$. Some distinctions are associated with the fact that the instruments were filled with different gas mixtures (various concentrations and pressures of ${ }^{3} \mathrm{He}$ and $\mathrm{CF}_{4}$ ).

\section{Conclusions}

A 2D detector for thermal neutrons has been successfully designed, constructed and tested. The following parameters have been achieved: active area $300 \times 170 \mathrm{~mm}^{2}$; a spatial resolution of $2.5 \mathrm{~mm}$ for the fine resolution axis and $4 \mathrm{~mm}$ for the discrete axis; efficiency about $60 \%$ (for a neutron wavelength of $9 \AA$ ).

To minimize detector outgasing, a new technology for the fabrication of the detector electrodes has been developed. All electrodes are fabricated from quartz-glass that can be pumped in high vacuum and heated up to $130^{\circ} \mathrm{C}$. Indeed, this opened a way to improve the gas purity by a few orders of magnitude. 
Currently, a new 2D neutron detector with an aperture of $300 \times 300 \mathrm{~mm}^{2}$ is under construction, where the anode wire spacing is $2 \mathrm{~mm}$ and the expected efficiency will be about $80 \%$ for $5-8 \AA$ neutrons.

\section{Acknowledgments}

We are very grateful to Prof. A. Okorokov for fruitful discussions on detector performance. We would like to thank I. Parchenko and N. Phlimonova for their strong contribution to the technology of detector production. We also thank the staff of PNPI workshop for approval and use of their facilities for detector production.

\section{References}

[1] V. Radeka, N.A. Schaknowski, G.C. Smith, B. Yu, Nucl. Instr. and Meth. A 419 (1998) 642.

[2] R.A. Boie, J. Fischer, Y. Inagaki, F.C. Merritt, H. Okuno, V. Radeka, Nucl. Instr. and Meth. 200 (1982) 533.

[3] R.B. Knott, G.C. Smith, G. Watt, J.W. Boldeman, Nucl. Instr. and Meth. A (392) (1997) 62. 